\title{
Tunable, large $X$-band microwave absorbers using variable chessboard surfaces
}

F. Ruiz-Perez, S. M. López-Estrada, F. Caballero-Briones

\begin{abstract}
In this communication, the design and electromagnetic absorption of a metamaterial coating with a broad modulable absorption in the 8 to $12 \mathrm{GHz}$ frequency range, corresponding to the $\mathrm{X}$-band, is reported. The unit of the coating material consists of a chessboard with chess squares of different heights made of a $\mathrm{rGO} / \mathrm{Fe}_{3} \mathrm{O}_{4} / \mathrm{PPy}$ composite. The influence of the height ratio of the chess squares in a $5 \times 5$ chessboard on the attenuation response of electromagnetic radiation in the $\mathrm{X}$ band was studied in silico. The results demonstrate that the chessboardlike microwave absorber exhibit tunable response, with a maximum effective attenuation of $-37.43 \mathrm{~dB} / \mathrm{GHz}$ in the $\mathrm{X}$ band range, ca. $24 \%$ higher than the best single layer coating.
\end{abstract}

Index Terms: Chessboard, composite, X-band, microwave absorber.

\section{Take-Home Messages:}

- In silico study of chessboard structure of $\mathrm{rGO} / \mathrm{Fe} 3 \mathrm{O}_{4} / \mathrm{PPy}$ as microwave absorber.

- The chessboard structure showed a tunable attenuation response in X-band region.

- Maximum reflection loss of $-35.83 \mathrm{~dB}$ and an effective attenuation in the X-band of -37.45 $\mathrm{dB} / \mathrm{GHz}$ was achieved.

\section{Introduction}

With the constant development of radar (Radio Detection and Ranging) systems, the investigation focusing on radar absorbing materials (RAM's) attracts increasing attention. Electromagnetic absorbers are a type of materials that absorb the incident electromagnetic Submission date: November $8^{\text {th }}, 2021$

The authors have no conflicts to disclose. This work was supported by SIP-IPN 20211513. F. Ruiz Perez is financed by CONACyT PhD and by BEIFI-IPN grants.

F. Ruiz-Perez and F. Caballero Briones are with the Instituto Politécnico Nacional, Materiales y Tecnologías para Energía, Salud y Medio Ambiente (GESMAT), CICATA Altamira. 89600, Altamira, México.

S. M. López Estrada is with the Unidad de Investigación y Desarrollo Tecnológico (UNINDETEC), SEMAR, 96269, Antón Lizardo, México.

Corresponding author: F. Caballero-Briones, e-mail: fcaballero@ipn.mx 
waves, attenuating the outcoming waves. These materials have important applications in areas such as invisibility cloaks, camouflage, stealth technology and electromagnetic shielding [1,2]. The development of RAM`s for the X-band (8-12 GHz) is of particular interest for civilian and military applications, for example, to ensure that tracking systems cannot detect targets in the area, prevent aerial interception. However, despite the large absorption peaks achieved in the literature, most of the studied metamaterials show a narrow absorption bandwidth, therefore, many efforts have been made to extend the bandwidth and cover wider bands of the electromagnetic spectrum [3-5]. For example, a $3 \mathrm{~mm}$ coating of a composite made of reduced graphene oxide ( $\mathrm{rGO})$, magnetite $\left(\mathrm{Fe}_{3} \mathrm{O}_{4}\right)$ and polypyrrole (PPy) has a reported maximum absorption of $-49.2 \mathrm{~dB}$ at $11.8 \mathrm{GHz}$, with a maximum effective absorption i.e., attenuation above $-10 \mathrm{~dB}$ in the studied band, of $-28.76 \mathrm{~dB} / \mathrm{GHz}$ [6]. Some strategies to increase the bandwidth are the use of multilayer materials [7,8], although they could suffer delamination due to interfacial stress during wearing. Other strategies include the use of metamaterials such as Au metallic metasurface arrays [9], chessboard-like metasurfaces with either alternating tiles [10] or square annular magnetic conductors, and Jerusalem cross structures [11]. However, the use of single layer, graphene-based absorbers in a metamaterial configuration have not been reported as wide band absorbers, to the best of our knowledge

In this letter, we report an in-silico study of the microwave attenuation response of a chessboard structure formed by squares of different heights made of the $\mathrm{rGO} / \mathrm{Fe} 3 \mathrm{O} 3 / \mathrm{PPy}$ composite, optimizing the height ratio to maximize the effective absorption in the X-band.

\section{Materials and Methods}

In the present study, the $\mathrm{rGO} / \mathrm{Fe}_{3} \mathrm{O}_{4} / \mathrm{PPy}$ composite reported by Zhang et al. [6] was used as coating material. The PPy and the addition of $\mathrm{Fe}_{3} \mathrm{O}_{4}$ adjust the permittivity and improve the impedance matching between the dielectric and the magnetic losses which allows the EM wave to penetrate the material and its subsequent dissipation as thermal energy. For the simulation reported here, Fig. 1a) shows a $5 \times 5$ chessboard-like surface composed by squares 
with two different heights which were varied as shown in the 1 . The simulation process was carried out using CST Studio software. The simulation parameters were set to obtain the reflectivity values in $\mathrm{dB}$ for varying the cells height in the frequency range 2-18 $\mathrm{GHz}$ [12]. The boundary conditions, material structure, and port configuration for the simulated measurement are shown in Fig. 1b). The simulated structure is composed of a perfect electric conductor (PEC) sheet as substrate, and as absorber, a single layer of the $\mathrm{rGO} / \mathrm{Fe}_{3} \mathrm{O}_{4} / \mathrm{PPy}$ composite reported in [11].

a)

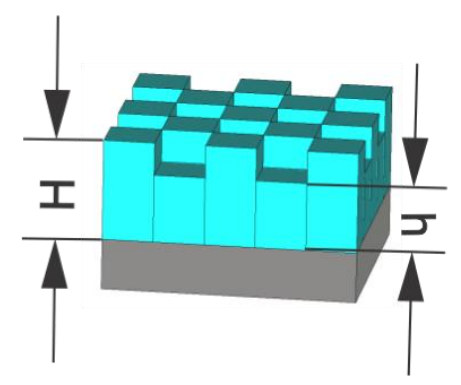

b)

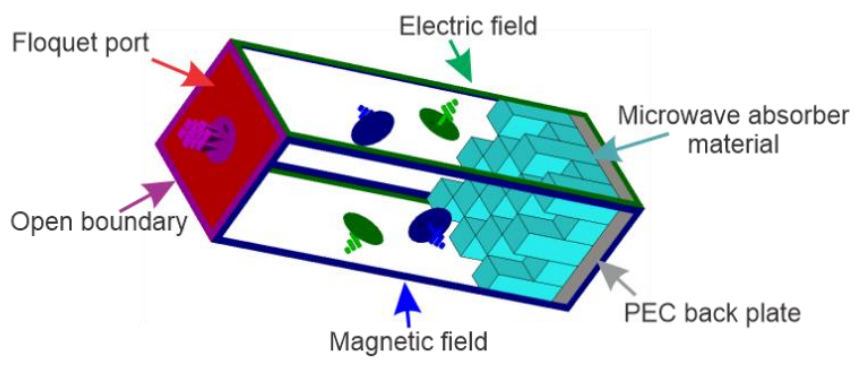

Fig. 1. a) Chessboard structure $\mathrm{rGO} / \mathrm{Fe}_{3} \mathrm{O}_{4} / \mathrm{PPy}$ composite. b) Setup used for simulation of proposed structure in CST studio.

Table 1. Height setup of the chess squares.

\begin{tabular}{ccc}
\hline $\mathbf{H}(\mathbf{m m})$ & $\mathbf{h}(\mathbf{m m})$ & $\mathbf{h} / \mathbf{H}$ \\
\hline 5 & 4 & 0.8 \\
5 & 2.8 & 0.56 \\
5 & 2.7 & 0.54 \\
5 & 2.5 & 0.5 \\
5 & 2.4 & 0.48 \\
5 & 2 & 0.4 \\
4 & 2.8 & 0.7 \\
4 & 2.7 & 0.675 \\
4 & 2.6 & 0.65 \\
4 & 2.5 & 0.625 \\
4 & 2.1 & 0.525 \\
\hline
\end{tabular}




\section{Results and Discussion}

Figure 2 presents the simulated attenuation response of the designed metamaterials in the 2$18 \mathrm{GHz}$ range. Figure 2a) presents single-layer, single thickness materials where the reported maximum absorption of $-37 \mathrm{~dB}$ at $11.8 \mathrm{GHz}$ for the optimized $\mathrm{rGO} / \mathrm{Fe} 3 \mathrm{O} / \mathrm{PPy}$ coating [11] is clearly observed. Despite the large absorption, the peak width is quite narrow. For comparison, in Figure 2b) the attenuation response of the designed metamaterials is presented. It can be observed that the maximum attenuation peak can be thoroughly modulated in the X-band, as well as the area under the curve.
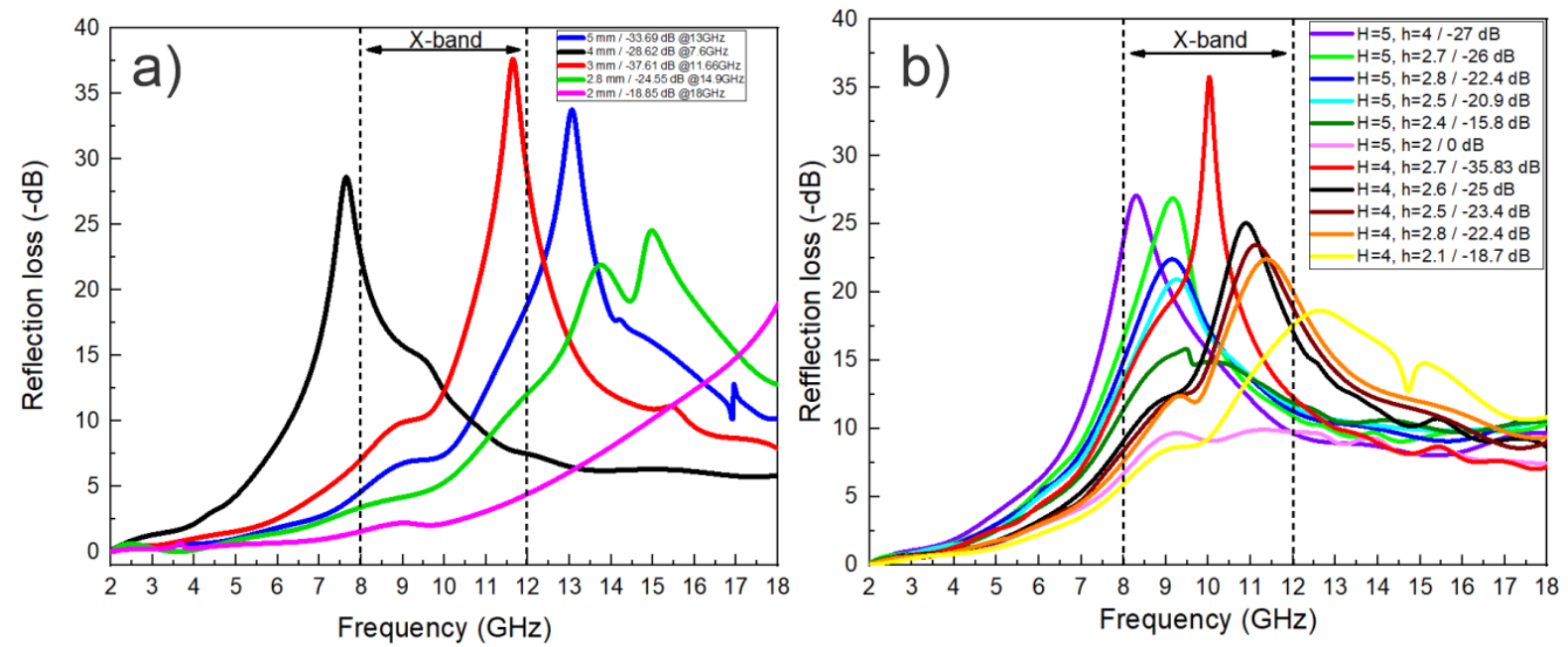

Fig 2. Attenuation responses of a) single layer, $\mathrm{rGO} / \mathrm{Fe}_{3} \mathrm{O}_{4} / \mathrm{PPy}$ composite with varying thickness; b) chessboard metamaterials with varying height ratios.

Figure 3 presents the effective attenuation in the $\mathrm{X}$-band, defined as the area under the curve where the reflection loss is higher than $-10 \mathrm{~dB}$ within the $8-12 \mathrm{GHz}$ range as shown in Figure 3a) inset. Figure 3a) presents the effective attenuation values of the single layer coatings. The effective attenuation reaches a maximum of $-28.77 \mathrm{~dB} / \mathrm{GHz}$ corresponding to a layer of 3 $\mathrm{mm}$; for layers with higher or lower thickness, the effective attenuation reduces abruptly. Figure $3 b$ ) presents the effective attenuation of the metamaterials based in chessboards constructed with $\mathrm{H}$ being $4 \mathrm{~mm}$ or $5 \mathrm{~mm}$ at the different $\mathrm{h} / \mathrm{H}$ ratios. The effective attenuation increases with the height ratio, i.e., as the difference between the highest $(\mathrm{H})$ and the shortest 
(h) square decreases. Limit effective attenuations were reached with $\mathrm{h} / \mathrm{H}$ ratios of 0.53 and 0.66 for $\mathrm{H}=4 \mathrm{~mm}$ and $\mathrm{H}=5 \mathrm{~mm}$, respectively. The chessboard configuration with $\mathrm{h}=2.7$ $\mathrm{mm}$ and $\mathrm{H}=4 \mathrm{~mm}$ has the largest effective attenuation with $-37.45 \mathrm{~dB} / \mathrm{GHz}$ within the $\mathrm{X}$ band, which corresponds to an increase of the total incident wave absorption of ca. $24 \%$ for the optimized chessboard configuration. After this in silico study, the preparation and experimental attenuation testing of the proposed coatings is intended.
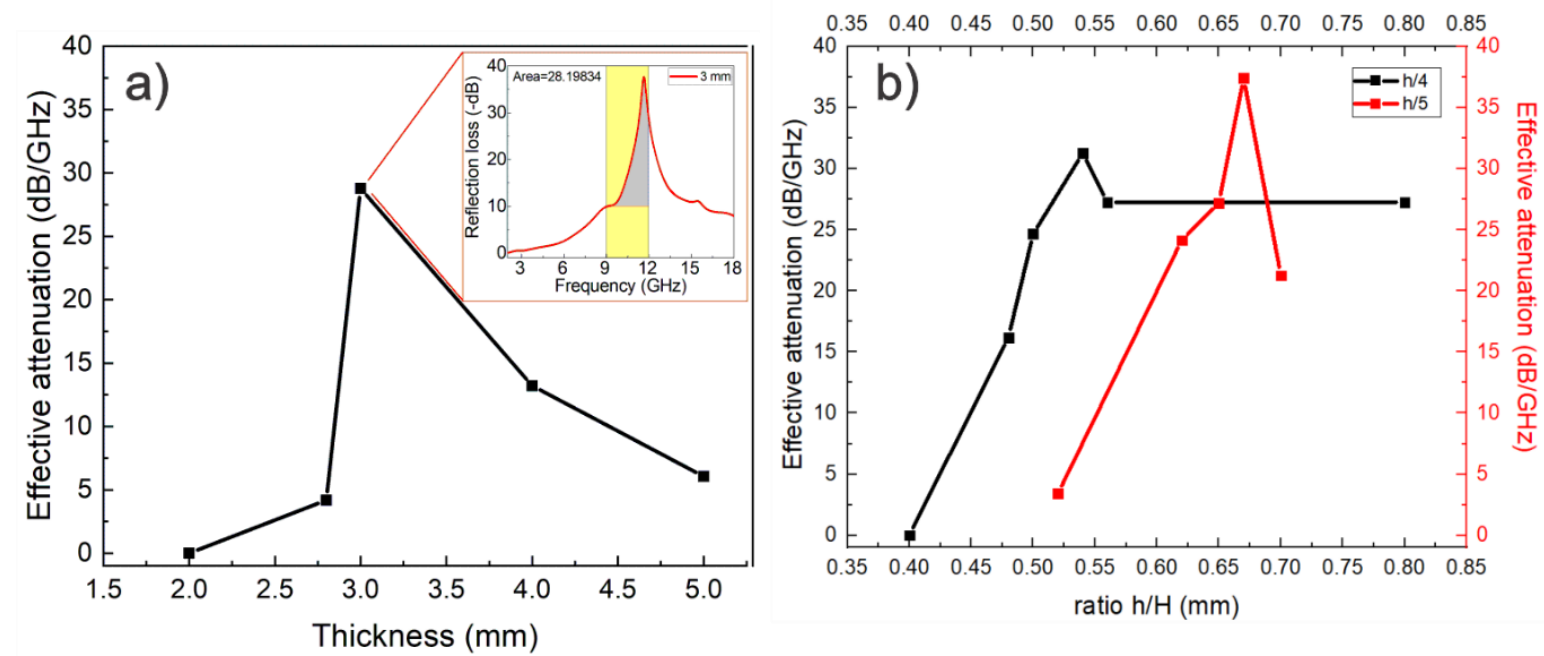

Fig 3. Effective attenuation in the $\mathrm{X}$-band $(8-12 \mathrm{GHz})$ of a) Single layer coating. b) Chessboard coating.

\section{Conclusions}

A single-layer chessboard structure with tunable absorption and large effective attenuation in the radar X-band, was investigated in silico. The simulated 5 x 5 chessboard was built by fixing the height of the "white" squares and varying the height of the "black" squares to study the effect of the height ratio on the effective attenuation of the structure in the $8-12 \mathrm{GHz}$ range. The squares were made of a $\mathrm{rGO} / \mathrm{Fe}_{3} \mathrm{O}_{4} / \mathrm{PANI}$ composite. The results showed a maximum attenuation value of $-35.83 \mathrm{~dB}$ at $10 \mathrm{GHz}$ with a height configuration of $\mathrm{H}=4 \mathrm{~mm}$ and $\mathrm{h}=2.7 \mathrm{~mm}$, with an effective bandwidth absorption, i.e., above $-10 \mathrm{~dB}$ in the $\mathrm{X}$-band range, of $-37.45 \mathrm{~dB} / \mathrm{GHz}$. In contrast with the response of single layer coatings, the chessboard structure allowed to modulate the attenuation frequency throughout the region of 
interest, as well as obtaining a wide band width. This study provides a first approach to further characterizations of the proposed structures in a real operative environment.

\section{CRediT author statement}

F. Ruiz-Perez: Conceptualization, Software, Investigation, Methodology, Validation, Visualization, Writing - Original Draft. S. M. López Estrada: Conceptualization, Supervision, Writing - Original Draft. F. Caballero-Briones: Conceptualization, Supervision, Formal analysis, Visualization, Writing - Original Draft.

\section{References}

[1] Islam SS, Hasan MM, Faruque MRI. A new metamaterial-based wideband rectangular invisibility cloak. Appl Phys A [Internet]. 2018;124:160. Available from: http://link.springer.com/10.1007/s00339-018-1590-9.

[2] Magisetty R, Raj AB, Datar S, et al. Nanocomposite engineered carbon fabric-mat as a passive metamaterial for stealth application. J Alloys Compd [Internet]. 2020;848:155771. Available from: https://linkinghub.elsevier.com/retrieve/pii/S0925838820321356.

[3] Handoko E, Budi S, Sugihartono I, et al. Microwave absorbing properties of teflon coating for $\mathrm{x}$-band frequencies. 2021. p. 030002. Available from: http://aip.scitation.org/doi/abs/10.1063/5.0037747.

[4] Di X, Wang Y, Fu Y, et al. Wheat flour-derived nanoporous carbon@ZnFe2O4 hierarchical composite as an outstanding microwave absorber. Carbon N Y [Internet]. 2021;173:174-184.

Available

from: https://linkinghub.elsevier.com/retrieve/pii/S0008622320310745.

[5] Zhang X, Liu Z, Deng B, et al. Honeycomb-like NiCo2O4@MnO2 nanosheets array/3D porous expanded graphite hybrids for high-performance microwave absorber with hydrophobic and flame-retardant functions. Chem Eng J [Internet]. 2021;419:129547. Available from: https://linkinghub.elsevier.com/retrieve/pii/S1385894721011347. 
[6] Zhang C, Chen Y, Li H, et al. Facile Fabrication of Three-Dimensional Lightweight RGO/PPy Nanotube/Fe 3 O 4 Aerogel with Excellent Electromagnetic Wave Absorption Properties. ACS Omega [Internet]. 2018;3:5735-5743. Available from: https://pubs.acs.org/doi/10.1021/acsomega.8b00414.

[7] Liu T, Kim S-S. Ultrawide Bandwidth Electromagnetic Wave Absorbers Using a High-capacitive Folded Spiral Frequency Selective Surface in a Multilayer Structure. Sci Rep [Internet]. 2019;9:16494. Available from: http://www.nature.com/articles/s41598-019-52967-z.

[8] Liu T, Kim S-S. Ultrawide Bandwidth Electromagnetic Wave Absorbers Composed of Double-Layer Frequency Selective Surfaces with Different Patterns. Sci Rep [Internet]. 2018;8:13889. Available from: http://www.nature.com/articles/s41598018-32181-z.

[9] Feng X, Xie X, Pu M, et al. Hierarchical metamaterials for laser-infrared-microwave compatible camouflage. Opt Express [Internet]. 2020;28:9445. Available from: https://www.osapublishing.org/abstract.cfm?URI=oe-28-7-9445.

[10] Haji-Ahmadi M-J, Nayyeri V, Soleimani M, et al. Pixelated Checkerboard Metasurface for Ultra-Wideband Radar Cross Section Reduction. Sci Rep [Internet]. 2017;7:11437. Available from: http://www.nature.com/articles/s41598-017-11714-y.

[11] Chen H, Shen R, Li F, et al. Design of phase matching chessboard-like electromagnetic metasurfaces for wideband radar cross section reduction. Microw Opt Technol Lett [Internet]. 2019;61:2037-2045. Available from: https://onlinelibrary.wiley.com/doi/10.1002/mop.31854.

[12] Lee YS, Wee FH, You KY, et al. Study of single layer microwave absorber based on rice husk Ash/CNTs composites. Indones J Electr Eng Comput Sci [Internet]. 2019;14:929. Available from: http://ijeecs.iaescore.com/index.php/IJEECS/article/view/16812. 